STRUCTURAL BIOLOGY COMMUNICATIONS

ISSN 2053-230X

Received 16 March 2015

Accepted 9 May 2015

Edited by H. M. Einspahr, Lawrenceville, USA

Keywords: nucleation; two-step mechanism; protein-rich clusters; crystallization.

\section{Do protein crystals nucleate within dense liquid clusters?}

\author{
Dominique Maes, ${ }^{\mathrm{a} *}$ Maria A. Vorontsova, ${ }^{\mathrm{b}}$ Marco A. C. Potenza, ${ }^{\mathrm{c}}$ Tiziano Sanvito, ${ }^{\mathrm{c}}$ \\ Mike Sleutel, ${ }^{a}$ Marzio Giglio ${ }^{c}$ and Peter G. Vekilov ${ }^{a, b, d}$
}

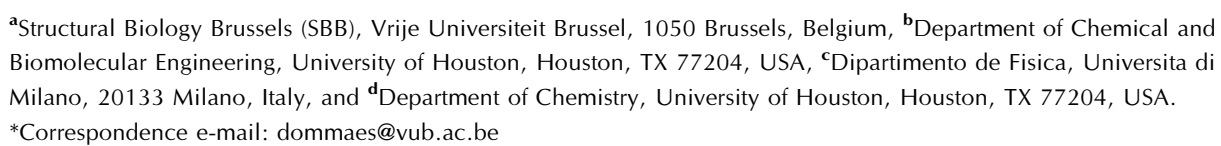

Protein-dense liquid clusters are regions of high protein concentration that have been observed in solutions of several proteins. The typical cluster size varies from several tens to several hundreds of nanometres and their volume fraction remains below $10^{-3}$ of the solution. According to the two-step mechanism of nucleation, the protein-rich clusters serve as locations for and precursors to the nucleation of protein crystals. While the two-step mechanism explained several unusual features of protein crystal nucleation kinetics, a direct observation of its validity for protein crystals has been lacking. Here, two independent observations of crystal nucleation with the proteins lysozyme and glucose isomerase are discussed. Firstly, the evolutions of the protein-rich clusters and nucleating crystals were characterized simultaneously by dynamic light scattering (DLS) and confocal depolarized dynamic light scattering (cDDLS), respectively. It is demonstrated that protein crystals appear following a significant delay after cluster formation. The cDDLS correlation functions follow a Gaussian decay, indicative of nondiffusive motion. A possible explanation is that the crystals are contained inside large clusters and are driven by the elasticity of the cluster surface. Secondly, depolarized oblique illumination dark-field microscopy reveals the evolution from liquid clusters without crystals to newly nucleated crystals contained in the clusters to grown crystals freely diffusing in the solution. Collectively, the observations indicate that the protein-rich clusters in lysozyme and glucose isomerase solutions are locations for crystal nucleation.

\section{Introduction}

Protein crystallization remains a challenge for structural biology and other areas relying on protein crystals (Chayen $e t$ al., 2010; Bergfors, 2009; McPherson, 2009). In many instances, the problems can be traced to the unpredictability of crystal nucleation. Nucleation is the first step of crystallization, which determines the availability of crystals and several crucial properties of the crystal population: polymorph selection, number of crystals, crystal sizes and size distribution, and others. Hence, fundamental understanding of protein crystal nucleation has been an active area of investigation (Akella $e t$ al., 2014; Arnold et al., 1999; Ataka \& Asai, 1990; Chayen, 2005; Chayen et al., 2006; D'Arcy et al., 2003; Dey et al., 2010; Feher \& Kam, 1985; Galkin \& Vekilov, 1999, 2000a,b, 2001; Haas \& Drenth, 2000; Lutsko et al., 2010; Malkin \& McPherson, 1994; Nanev \& Penkova, 2001; Nicolis \& Maes, 2012; Pan et al., 2005; Pechkova \& Nicolini, 2002; Penkova et 
al., 2006; Reguera \& Rubí, 2003; Sear, 2009; Selimović et al., 2009; Shiryayev \& Gunton, 2004; Sleutel et al., 2014; Talanquer, 2005; ten Wolde \& Frenkel, 1997; Tsekova et al., 2002; Vekilov, 2004, 2005, 2010a; Yau \& Vekilov, 2001).

One of the significant recent findings in this pursuit has been the formulation of the two-step mechanism of nucleation (Galkin \& Vekilov, 2000b; Vekilov, 2004, 2010a; Vekilov \& Vorontsova, 2014; Chung et al., 2010; Sauter et al., 2015; Sleutel \& Van Driessche, 2014). According to this mechanism, the nucleation of crystals is preceded by the formation of clusters of mesoscopic size composed of dense protein liquid (Gliko et al., 2005a, 2007; Pan et al., 2007, 2010; Vekilov et al., 2008). Crystal nuclei then form inside the clusters (Galkin \& Vekilov, 2000b; Garetz et al., 2002; Vekilov, 2004). Protein-rich clusters that may be the nucleation precursors have been observed in solutions of several proteins: lysozyme, haemoglobin A and S, lumazine synthase, insulin and others. The size of the clusters typically varies from several tens to several hundreds of nanometres and their total volume fraction remains less than $10^{-3}$. It was shown that these clusters are present even in the homogeneous region of protein phase diagrams (Gliko et al., 2005a, 2007; Pan et al., 2007, 2010; Li et al., 2012) and their extended lifetimes indicate that they are not concentration fluctuations.

Direct imaging of crystal nuclei forming within dense liquid clusters has been provided for two types of systems: colloids, which are larger and move more slowly than most molecules (Savage \& Dinsmore, 2009), and an ingeniously chosen organic system (Harano et al., 2012). The evidence for the action of this two-step mechanism in the formation of nuclei of protein crystals (Vekilov, 2010a; Sauter et al., 2015), sickle-cell anaemia fibres (Galkin et al., 2007) and amyloid fibrils (Lomakin et al., 1996; Krishnan \& Lindquist, 2005) has mostly been indirect: the two-step mechanism was put forth to explain unusual nonmonotonic dependencies of the protein crystal nucleation rate on supersaturation and a tenfold orderof-magnitude discrepancy between the nucleation rates predicted by the classical nucleation theory assuming one-step crystal nucleation and the actual data (Vekilov, 2004, 2010a).

Nucleation of protein crystals and other ordered solids (e.g. sickle haemoglobin fibres) in stable dense protein liquid has been observed numerous times (Galkin et al., 2002; Vivarès et al., 2005). Direct observation of protein crystal nucleation inside the metastable clusters is challenging owing to a protein cluster size which typically is below the optical resolution limit. To tackle this challenge, in this work we monitored the initial progress of crystallization using two novel techniques: confocal depolarized dynamic light scattering (cDDLS) and depolarized oblique illumination dark-field microscopy (DOIDM). Monitoring of supersaturated protein solutions by depolarized optics allows the direct detection of crystals shortly after their formation. We demonstrate that the clusters are liquid in nature and that crystals always nucleate after clusters. We show that the motions of the nucleated crystals are not diffusive. We directly detect newly nucleated crystals within protein-rich clusters. We employed lysozyme for the cDDLS studies and glucose isomerase for the DOIDM tests.

\section{Materials and methods}

\subsection{The protein solutions}

Two stock solutions of hen egg-white lysozyme (Affymetrix, USA) were prepared: at a concentration of $138 \mathrm{mg} \mathrm{ml}^{-1}$ in $20 \mathrm{~m} M$ HEPES ( $N$-2-hydroxyethylpiperazine- $N$-2-ethanesulfonic acid) buffer $\mathrm{pH} 7.8$ and at a concentration of $157 \mathrm{mg} \mathrm{ml}^{-1}$ in $50 \mathrm{~m} M$ sodium acetate buffer $\mathrm{pH}$ 4.5. Glucose isomerase from Streptomyces rubiginosus (Hampton Research, USA) was dialyzed against $100 \mathrm{~m} M$ HEPES buffer

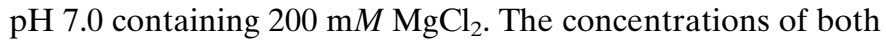
proteins were determined by UV absorbance at $280 \mathrm{~nm}$ using extinction coefficients of $2.64 \mathrm{ml} \mathrm{mg}^{-1} \mathrm{~cm}^{-1}$ for lysozyme and $1.042 \mathrm{ml} \mathrm{mg}^{-1} \mathrm{~cm}^{-1}$ for glucose isomerase. All stock solutions were filtered through $0.22 \mu \mathrm{m}$ syringe filters after preparation. For the crystallization experiments, supersaturation was achieved by adding sodium chloride and ammonium sulfate to the lysozyme and glucose isomerase solutions, respectively. All experiments were carried out at $22^{\circ} \mathrm{C}$.

\subsection{Confocal depolarized dynamic light scattering (cDDLS) in combination with dynamic light scattering (DLS)}

If a collection of birefringent scatterers is illuminated by a vertically polarized electric field $E_{\mathrm{V}}$, the scattered field can be described by two components, $E_{\mathrm{VV}}$ and $E_{\mathrm{VH}}$. The polarized $E_{\mathrm{VV}}$ is significantly more intense than the depolarized $E_{\mathrm{VH}}$ owing to the small birefringent power of the scatterers. When the scattered field is superimposed with the transmitted field, the scattered signal is heterodyned by the transmitted field, acting as a local oscillator. Unfortunately, since the scattered field is in quadrature with the transmitted field, this interference does not affect the intensity of the forward beam. To overcome this problem, traditionally a quarter-wave plate was inserted before the analyzer, so that only the scattered beam is phase-shifted by $90^{\circ}$, leading to a destructive interference affecting the measured intensity. We chose a different arrangement. We expanded the scattering amplitudes for birefringent scatterers, which promoted the second-order term to a quadrature of phase with the transmitted beam. The time fluctuations of both terms are synchronous. Therefore, no need arises for a quarter-wave plate: the amplitude of the second term is sufficiently large. We found that a confocal geometry, as described in Potenza et al. (2010, 2012), was the breakthrough: a strong second-order term ultimately requires a strong scattering efficiency, which leads to multiple scattering, the greatest limitation of the DDLS technique in the past. The cDDLS intensity scales with the anisotropy of the polarizability of the crystals, the number/size of crystals in the scattering volume and the relative refractive index of the crystals and the solution. Importantly, if all scatterers in the monitored volume are isotropic, no fluctuations are expected from the light collected in the forward direction.

The experimental setup is shown in Fig. 1(a). A spatially filtered, collimated He-Ne laser beam $(\lambda=632.8 \mathrm{~nm}, 30 \mathrm{~mW})$ passes through a Glan-Thompson polarizer $(\mathrm{P})$ with vertical transmission. The beam is focused on the experimental cell volume (four optical windows, $2 \mathrm{~mm}$ optical path) via a 
microscope objective lens (Nachet $20 \times$, numerical aperture 0.3 ). The DLS signal is collected at $90^{\circ}$ by a lens (L2) and transmitted via a monomode optical fibre connected to a photomultiplier tube (PMT). For the cDDLS measurements the transmitted beam is sent to a collection optics identical to a confocal scheme where light is only collected from the volume illuminated by the focused beam. The forward scattered and the transmitted light are sent through an analyzer (A) at almost complete extinction, so that the whole depolarized signal can be superimposed on a small fraction of the transmitted beam projected in the horizontal direction. A second PMT collects the forward propagating light through a monomode optical fibre. The DLS and cDDLS signals from the

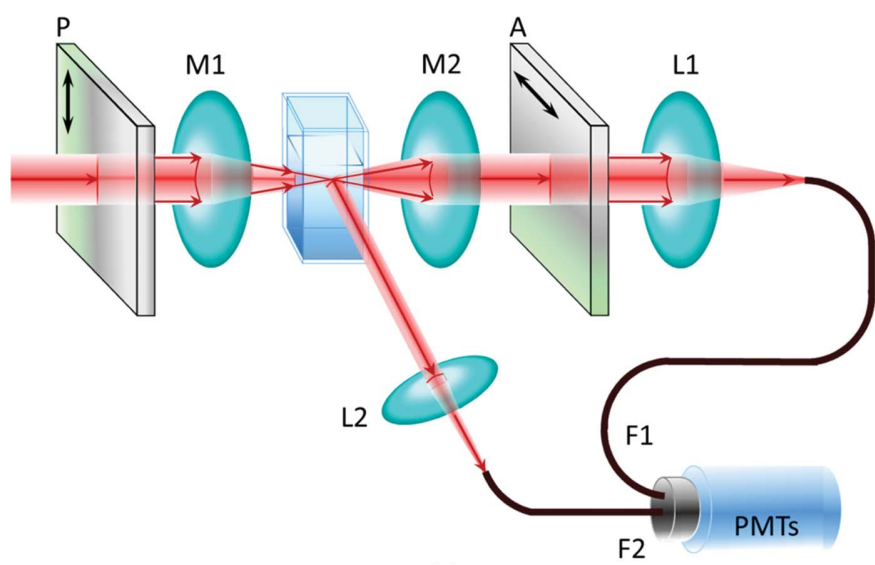

(a)

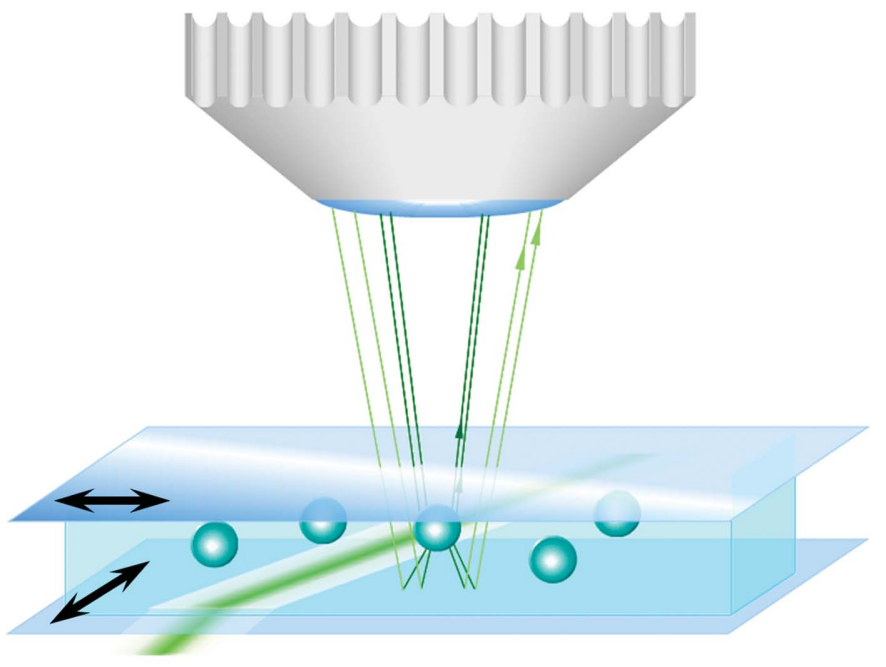

(b)

Figure 1

Schematic drawings of the experimental devices. (a) The experimental setup for combined dynamic light scattering (DLS) and confocal depolarized dynamic light scattering (cDDLS). A laser beam is polarized by the polarizer P and focused by lens M1 into the sample cell. Light scattered at $90^{\circ}$ is introduced by lens L2 to an optical fibre F2 and transmitted to a photomultiplier (PMT). The transmitted light passing through an analyzer $\mathrm{A}$ at complete extinction with $\mathrm{P}$ is collected by lens L1 in a confocal scheme and sent by optical fibre F1 to a second photomultiplier. Both PMTs are connected to correlators. (b) Depolarized oblique illumination dark-field microscopy (DOIDM) setup. A laser beam illuminates a $\sim 100 \mu \mathrm{m}$ wide path in a rectangular sample cell. The light scattered vertically is collected by an objective lens. A polarizer and analyzer (indicated by black arrows) are placed at the optical entrance and exit of the sample cell, respectively. respective photomultipliers are analyzed with digital multi-tau correlators, resulting in the DLS intensity and the cDDLS intensity autocorrelation functions. Details of the method are given in Potenza et al. $(2010,2012)$.

The cDDLS and DLS intensity autocorrelation functions $g_{2}$ were acquired for $60 \mathrm{~s}$. As in numerous previous investigations, the DLS correlation functions revealed the presence of two scatterers with distinct diffusion times: $\tau_{1}$ and $\tau_{2}$ (Gliko et al., 2005a, 2007; Pan et al., 2007, 2010; Li et al., 2011, 2012; Sleutel \& Van Driessche, 2014; Vekilov \& Vorontsova, 2014). In analogy to previous work, $\tau_{1}$ was assigned to the protein monomers, while $\tau_{2}$ was assigned to clusters. To determine $\tau_{1}$ and $\tau_{2}$ the DLS data were analyzed as described in Li et al. (2011) using the fitting function

$$
\left[g_{2}(t)-1\right]^{1 / 2}=A_{1} \exp \left(-\frac{t}{\tau_{1}}\right)+A_{2} \exp \left(-\frac{t}{\tau_{2}}\right)+b,
$$

where $A_{1}$ and $A_{2}$ are their respective relative amplitudes; $b$ is a baseline correction.

The cluster radius was determined from $\tau_{2}$ using the Stokes-Einstein relation; for details, see Pan et al. (2007) and Li et al. (2011). The viscosity of the solutions needed to evaluate the cluster radius was determined independently as described in Pan et al. (2007), Li et al. (2011) and Sleutel et al. (2012) by monitoring the diffusion of polystyrene spherical particles with diameter $600 \mathrm{~nm}$.

The cDDLS data of interest (the interval between 0.001 and $2 \mathrm{~s})$ were first smoothed by convolution with a normalized rect function. Two functions were fitted to the data: a single exponential function

$$
\left[g_{2}(t)-1\right]^{1 / 2}=A \exp \left(-\frac{t}{\tau}\right)+b
$$

and a Gaussian function

$$
\left[g_{2}(t)-1\right]^{1 / 2}=\frac{A}{(2 \pi)^{1 / 2} \sigma} \exp \left(\frac{-t^{2}}{2 \sigma^{2}}\right)+b .
$$

To evaluate the sensitivity of the method, we followed the discussion in Potenza et al. (2012). We calculated the scattering field matrix of a lysozyme crystal with an effective radius of $300 \mathrm{~nm}$. We used the Amsterdam discrete-dipole approximation code, which is particularly suitable for this kind of numerical computation (Yurkin \& Hoekstra, 2011). We obtained that the component of the adimensional field amplitude contributing to the cDDLS signal is of the order of $4 \times 10^{-5}$. We compare this value with that obtained for a monodisperse suspension of MFA spheres $95 \mathrm{~nm}$ in diameter, which present a crystallinity of approximately $30 \%$, as used for method validation in Potenza et al. $(2010,2012)$. In that earlier case, the same component of the adimensional field is less than $10^{-6}$. Still, particle size could be accurately determined with only $N=10$ particles simultaneously present in the scattering volume. Note that owing to the heterodyne conditions of cDDLS, the random superposition of the field scales as $N^{1 / 2}$ (Potenza et al., 2012). Thus, the resulting value for the field amplitude is smaller than $3 \times 10^{-6}$, which is one order of magnitude less than we expect for a lysozyme crystal. These 

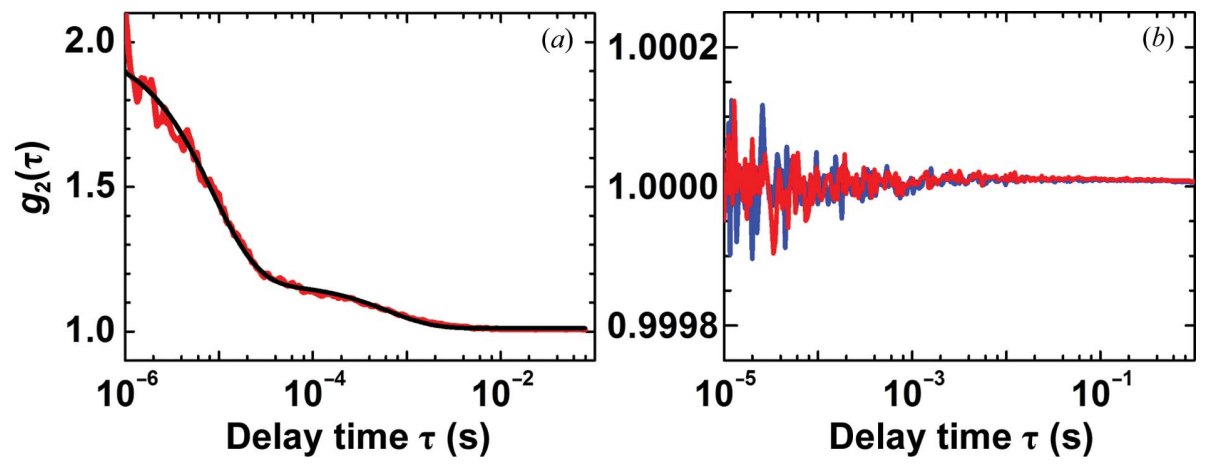

Figure 2

(a) A DLS intensity correlation function (red) of a lysozyme solution at $138 \mathrm{mg} \mathrm{ml}^{-1}$ in $20 \mathrm{mM}$ HEPES buffer $\mathrm{pH} 7.8$ and the fitted function (black). (b) cDDLS intensity correlation functions of the same lysozyme solution (red) and water (blue).
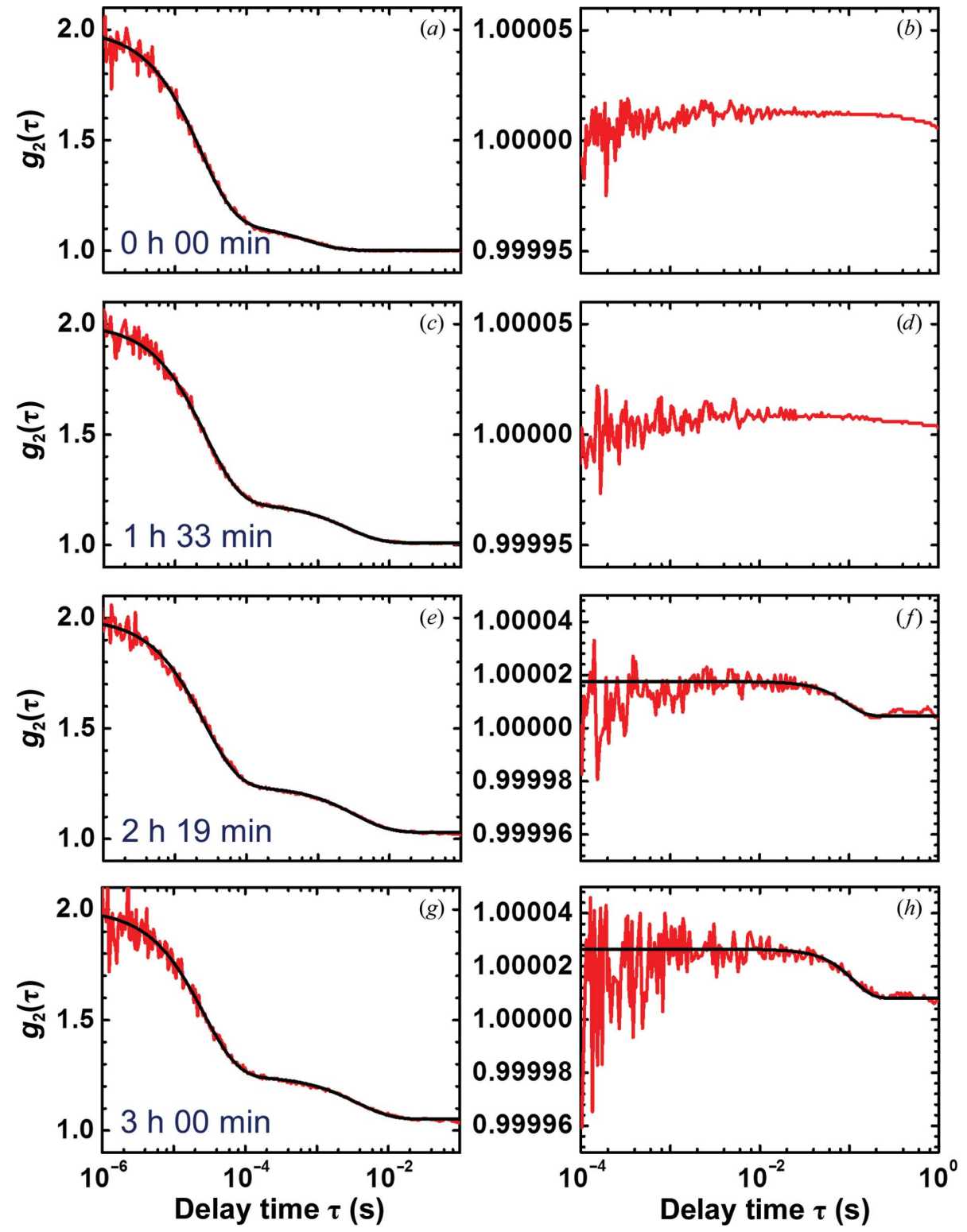

Figure 3

DLS (left column) and cDDLS (right column) intensity correlation functions recorded in parallel from the same lysozyme solution $\left[50 \mathrm{mg} \mathrm{ml}^{-1}\right.$ in $100 \mathrm{~m} M$ sodium acetate buffer $\mathrm{pH} 4.5$ with $30 \mathrm{mg} \mathrm{ml}^{-1}(0.51 \mathrm{M})$ sodium chloride] at the times after onset of crystallization indicated in the plots. Before the experiment, all components of the mixture, water, buffer, salt and protein stock solution, were characterized separately. No cDDLS signal was detected. The fitted curves are indicated in black. 


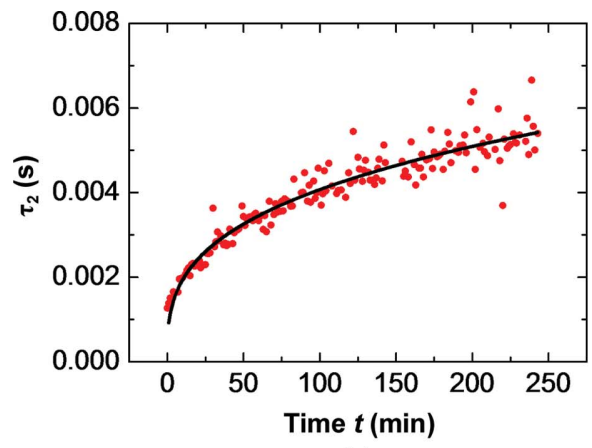

(a)

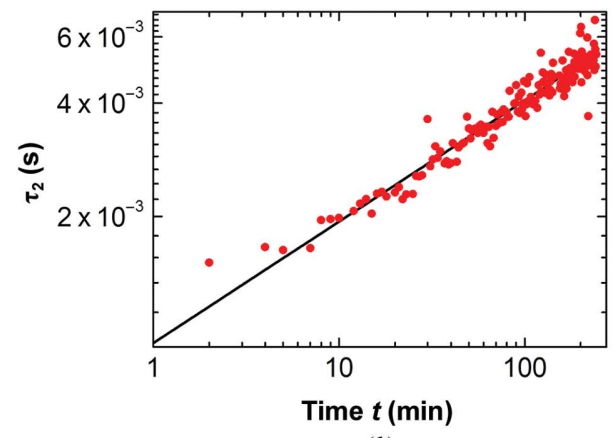

(b)

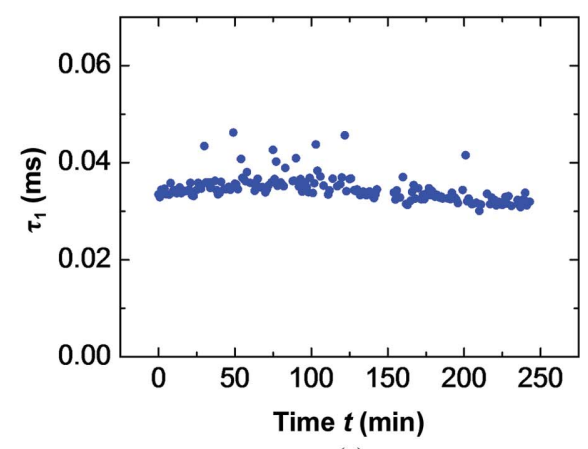

(c)

The evolution of the characteristic diffusion times of the clusters $\tau_{2}$ in $(a)$ and $(b)$ and monomers $\tau_{1}$ in $(c)$ extracted from DLS correlation functions similar to those in Figs. 3(a), 3(c), 3(e) and 3(g) during the crystallization of lysozyme $\left[50 \mathrm{mg} \mathrm{ml}^{-1}\right.$ in $100 \mathrm{~m} M$ sodium acetate buffer pH 4.5 with $30 \mathrm{mg} \mathrm{ml}^{-1}(0.51 \mathrm{M})$ sodium chloride]. The time $t$ is measured from the onset of the experiment. The black line in $(a)$ and $(b)$ depicts the $t^{0.32}$ dependence, which is close to the $t^{0.33}$ typical of Ostwald ripening.

estimates indicate that the cDDLS method is sensitive to single lysozyme crystals.

\subsection{Depolarized oblique illumination dark-field microscopy (DOIDM)}

A Nanosight LM10-HS microscope (Nanosight Ltd) equipped with a green laser (wavelength $532 \mathrm{~nm}$ ) was employed to monitor individual clusters in the tested solutions (Fig. 1b). The raw data of this method are movies of pointspread functions of clusters undergoing Brownian motion. To detect crystals, we modified the commercial setup by adding a polarizer at the optical entrance to the sample cell and an analyzer at the optical exit before the objective lens.

\section{Results and discussion}

\subsection{The protein-rich clusters}

We simultaneously measured DLS and cDDLS intensity correlation functions of a lysozyme solution in undersaturated conditions (138 $\mathrm{mg} \mathrm{ml}^{-1}, 20 \mathrm{~m} M$ HEPES pH 7.8) where no crystals can nucleate (Fig. 2). Analyses of the DLS correlation functions revealed the presence of lysozyme monomers and clusters with characteristic diffusion times $\tau_{1}=0.012 \mathrm{~ms}$ and $\tau_{2}$ $=1 \mathrm{~ms}$, respectively. Using the Stokes-Einstein relation and accounting for the viscosities of the buffer, which scales the monomer radius, and of the protein solution, which scales the cluster radius, the corresponding hydrodynamic radii of these two scatterers are 1.5 and $43 \mathrm{~nm}$, respectively. The monomer radius is close to the value determined from the atomic structure of the lysozyme molecule (Wang et al., 2007). The mean cluster radius is consistent with that previously found under similar conditions (Li et al., 2012; Pan et al., 2010). The cDDLS correlation data from the protein sample are identical to those of water, indicating that the protein-rich clusters are liquid or amorphous solid objects.

\subsection{Crystal nucleation within protein-rich clusters detected with cDDLS}

To correlate the cluster characteristics with the nucleation of crystals, we simultaneously recorded cDDLS and DLS intensity correlation functions at regular time intervals after the preparation of a lysozyme solution supersaturated with respect to crystals (Fig. 3). The crystallization process was initiated by adding sodium chloride to a lysozyme solution in acetate buffer. Data collection was started immediately after mixing. The DLS correlation functions (several examples are displayed in Figs. $3 a, 3 c, 3 e$ and $3 g$ ) revealed the presence of clusters from the onset of the experiment. The characteristic diffusion times of the clusters, determined from the entire sequence of correlation functions and displayed in Figs. 4(a) and 4(b), reveal that the cluster size increases in time $t$ as $t^{0.32}$, while the characteristic diffusion time of the monomers $\tau_{1}$ in Fig. $4(c)$ is steady. The likely reason for the $\tau_{2}$ evolution is Ostwald ripening of the clusters, as observed for lysozyme clusters under similar conditions by Li et al. (2012). Ostwald ripening is driven by the minimization of the surface free energy of the cluster population (Ostwald, 1896; Lifshitz \& Slyozov, 1961) so that smaller clusters disappear, while larger clusters grow on behalf of the released protein, resulting in growth of the mean cluster size and characteristic diffusion time $\tau_{2}$.

The correlation functions of the depolarized cDDLS signal, sampled in Figs. $3(b), 3(d), 3(f)$ and $3(h)$, reveal the appearance of a shoulder with characteristic time $\sim 0.1 \mathrm{~s}$ after approximately $140 \mathrm{~min}$ (Figs. $3 f$ and $3 h$ ). This shoulder indicates the presence of crystals in the cDDLS scattering volume. The cDDLS signal deviates from an exponential decay and is surprisingly well fitted with a Gaussian function (Fig. 5). The faster than exponential decay of the correlation function excludes the possibility that it reflects the polydispersity of the crystal population: polydispersity would have resulted in a stretched exponential decay. The non-exponential decay could be explained by assuming that the crystals participate in a nondiffusive motion. Three nondiffusive motions are possible: (i) the crystals are carried across the beam by convection owing to thermal gradients in the cell, (ii) the crystals are carried across the beam by sedimentation in the gravity field or (iii) the crystals are contained within dense liquid clusters that change shape.

To test scenarios (i) and (ii), we carefully analyzed the time sequence of the signal processed by the correlator. The char- 
acteristic time of decorrelation of the cDDLS signal in Figs. $3(f)$ and $3(h), 0.1 \mathrm{~s}$, would require the crystal to pass through the cDDLS sampling width of $1 \mu \mathrm{m}$ with a constant velocity of about $10 \mu \mathrm{m} \mathrm{s}^{-1}$. With respect to scenario (i) we note that at the very start of nucleation, where the concentration gradients are minor, convection could only be driven by thermal gradients and its velocity would be of the order of $0.1-1 \mu \mathrm{m} \mathrm{s}^{-1}$ (Lin et al., 1995, 2001). This refutes scenario (i).

Concerning scenario (ii), in which the motion of the crystal is owing to sedimentation, the upper bound value of the difference in density between tetragonal lysozyme crystals (that form in the presence of sodium chloride) and the solution is $\sim 0.3 \mathrm{~g} \mathrm{~cm}^{-3}$ (Quillin \& Matthews, 2000). From this, sedimentation velocities of the order of $10 \mu \mathrm{m} \mathrm{s}^{-1}$ would be attained by crystals of size $10 \mu \mathrm{m}$ or larger. Such crystals would perturb the DLS signal and be detectable in it. Such perturbations are absent in the DLS correlation functions in Figs. 3(e) and 3(g).

Thus, scenario (iii), in which the crystals are contained within dense liquid clusters and are driven by the elastic response of the cluster surfaces to Brownian collisions with the

Figure 5

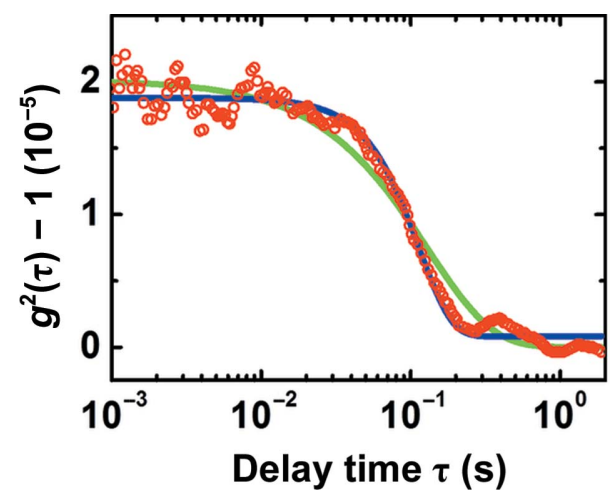

A typical cDDLS intensity correlation function (red symbols). Green line, best fit to data with an exponential decay function. Blue line, best fit to data with a Gaussian decay function. solvent molecules, is a feasible explanation. As a first step in testing scenario (iii), below we explore the liquid nature of the clusters.

\subsection{DOIDM characterization of clusters and crystal nucleation within clusters}

We tested the liquid nature of the protein-rich clusters suggested by previous indirect evidence (Gliko et al., 2005b, 2007; Pan et al., 2007) with aged solutions of glucose isomerase, which hold protein-rich clusters of relatively large size $(0.5-1.0 \mu \mathrm{m})$. Oblique illumination dark-field microscopy performed without a polarizer and analyzer exhibits unique intensity patterns that are readily distinguishable from those of solid protein aggregates, similar to those in Fig. $6(a)$. The patterns are dynamic and highly asymmetrical, with interference fringes spreading from the centre. The orientation of these fringes and their intensity vary with time. The minimum of the surface free energy for a liquid cluster corresponds to a spherical shape. However, Brownian collisions with the solvent molecules lead to deviations from this shape, resulting in the highly asymmetric dynamics of the intensity pattern. We conclude that the clusters are liquid and the surface free energy between the cluster and the solution is too low to stabilize a steady sphere. No determinations of the surface free energy of the protein-rich clusters have been performed. However, the free surface energy of dense liquid droplets in lysozyme solutions has been estimated as $\gamma=4 \times 10^{-5} \mathrm{~J} \mathrm{~m}^{-2}$ (Vekilov, 2010b). With this $\gamma$, the excess free energy of a protrusion of radius $r=100 \mathrm{~nm}$ can be estimated as $\Omega \gamma / r \simeq$ $10^{-23} \mathrm{~J}\left(\Omega=3 \times 10^{-26} \mathrm{~m}^{-3}\right.$ is the molecular volume). This is significantly less than the driving force for shape change, the thermal energy of the solvent molecules $k_{B} T \simeq 10^{-21} \mathrm{~J}$ (where $k_{\mathrm{B}}$ is the Boltzmann constant and $T$ is the temperature). Assuming that the surface free energy of the protein-rich clusters in glucose isomerase solutions is similar to this value, shape dynamics in response to Brownian collisions with the

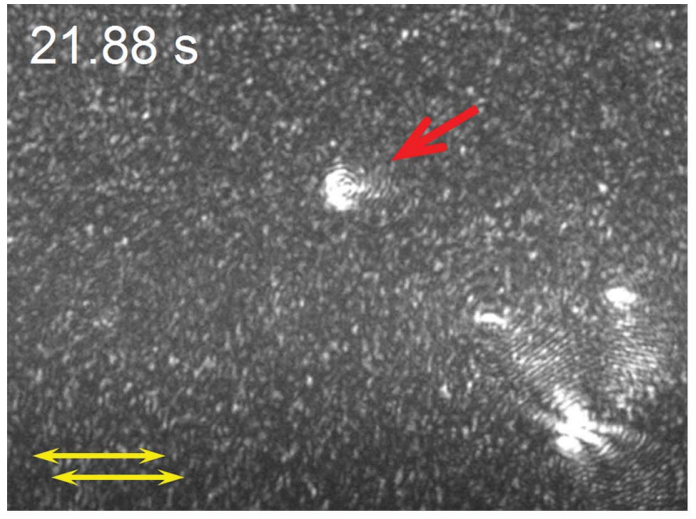

(a)

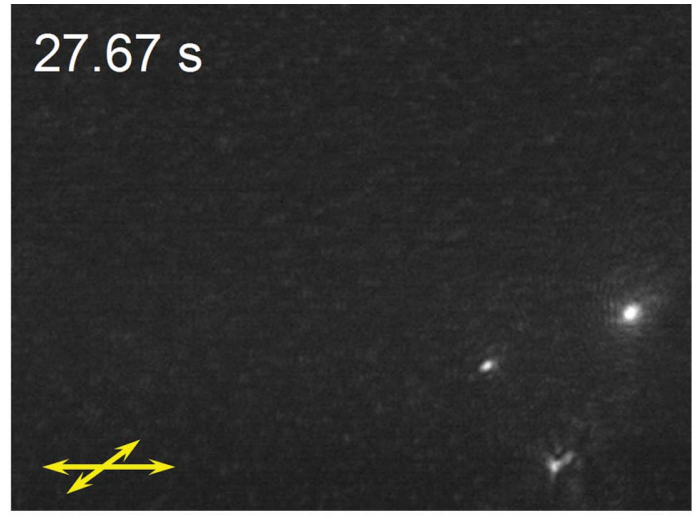

(b)

Figure 6

Depolarized oblique illumination dark-field microscopy (DOIDM) of a supersaturated glucose isomerase solution $\left(90 \mathrm{mg} \mathrm{ml} l^{-1}\right.$ in $100 \mathrm{~m} M \mathrm{HEPES}$ buffer $\mathrm{pH} 7.0$ with $200 \mathrm{mM} \mathrm{MgCl}$ and $1 M$ ammonium sulfate). The two images were taken $\sim 40$ min after the onset of the crystallization process in (a) parallel and $(b)$ crossed polarizers. The time shown in the images is measured after the beginning of video recording. Judging from their changing shapes the intensity peaks in $(a)$ are clusters. The cluster indicated by a red arrow in $(a)$ is not seen in $(b)$. The three intensity peaks in the bottom right corner of $(a)$ are also seen in $(b)$, suggesting that they represent small crystals within clusters. The time interval between the images is owing to the adjustment of the analyzer. 
solvent is feasible and the glucose isomerase clusters are liquid. If we transfer the conclusion of a liquid nature of the clusters to the clusters in lysozyme solutions, we provide the last missing link that attributes the nondiffusive cDDLS signal to crystals contained within clusters with liquid dynamic shape.

To study the nucleation of crystals, we added ammonium sulfate to glucose isomerase solution and loaded the solution into the DOIDM cell. If the polarizer and analyzer were in a parallel orientation we detected bright spots corresponding to individual clusters immediately after solution loading; a perpendicular arrangement of these two optical elements resulted in completely dark images. This indicates that only amorphous liquid clusters are present in the tested solutions. 40 min after the beginning of an experiment we see bright intensity spots with a crossed polarizer and analyzer, indicating the presence of objects that rotate the plane of polarization. Solution filtration prior to loading removed all stray crystals from the monitored volume; hence, we conclude that the only particles that may depolarize light are protein crystals. This signal indicates protein crystal nucleation. Further observations revealed three types of particles in the solution: (i) those visible only with a parallel polarizer and analyzer with a cluster-like intensity pattern, i.e. large protein clusters; (ii) those visible with both a parallel and a crossed polarizer and analyzer but with a steady shape; we conclude that these are large freely diffusing crystals; and (iii) particles that are visible with both a parallel and a crossed polarizer and analyzer but with a cluster-like pattern in parallel mode and a crystal-like pattern in crossed mode. We conclude that these are protein clusters with entrapped protein crystals inside.

In Fig. 6 we show two snapshots from a DOIDM movie, where we indicate the position of the analyzer and polarizer and the times corresponding to these frames. Fig. $6(a)$ is an example of the intensity patterns typically observed for large clusters in parallel polarizers. Fig. $6(b)$ shows three crystals in crossed polarizers seen as a steady bright spot. Comparing the locations of the crystals and three of the clusters, we conclude that these are most probably protein clusters with entrapped protein crystals inside.

\section{Acknowledgements}

PGV gratefully acknowledges the support of the Francqui Foundation for his stay as a Francqui International Professor at VUB. Funding for this work was provided by BelSPO (ESA-Prodex AO-2004-070) to DM and by NSF (grant MCB-1244568) and NASA (grants NNX14AE79G and NNX14AD68G) to PGV.

\section{References}

Akella, S. V., Mowitz, A., Heymann, M. \& Fraden, S. (2014). Cryst. Growth Des. 14, 4487-4509.

Arnold, S., Goddard, N. L. \& Wotherspoon, N. (1999). Rev. Sci. Instrum. 70, 1473-1477.

Ataka, M. \& Asai, M. (1990). Biophys. J. 58, 807-811.

Bergfors, T. (2009). Protein Crystallization, 2nd ed. La Jolla: International University Line.

Chayen, N. E. (2005). Prog. Biophys. Mol. Biol. 88, 329-337.
Chayen, N. E., Helliwell, J. R. \& Snell, E. H. (2010). Macromolecular Crystallization and Crystal Perfection, p. 24. Oxford University Press.

Chayen, N. E., Saridakis, E. \& Sear, R. P. (2006). Proc. Natl Acad. Sci. USA, 103, 597-601.

Chung, S., Shin, S.-H., Bertozzi, C. R. \& De Yoreo, J. J. (2010). Proc. Natl Acad. Sci. USA, 107, 16536-16541.

D'Arcy, A., Mac Sweeney, A. \& Haber, A. (2003). Acta Cryst. D59, 1343-1346.

Dey, A., Bomans, P. H. H., Müller, F. A., Will, J., Frederik, P. M., de With, G. \& Sommerdijk, N. A. J. M. (2010). Nature Mater. 9, 10101014.

Feher, G. \& Kam, Z. (1985). Methods Enzymol. 114, 77-112.

Galkin, O., Chen, K., Nagel, R. L., Hirsch, R. E. \& Vekilov, P. G. (2002). Proc. Natl Acad. Sci. USA, 99, 8479-8483.

Galkin, O., Pan, W., Filobelo, L., Hirsch, R. E., Nagel, R. L. \& Vekilov, P. G. (2007). Biophys. J. 93, 902-913.

Galkin, O. \& Vekilov, P. G. (1999). J. Phys. Chem. B, 103, 1096510971.

Galkin, O. \& Vekilov, P. G. (2000a). J. Am. Chem. Soc. 122, 156-163.

Galkin, O. \& Vekilov, P. G. (2000b). Proc. Natl Acad. Sci. USA, 97, 6277-6281.

Galkin, O. \& Vekilov, P. G. (2001). J. Cryst. Growth, 232, 63-76.

Garetz, B., Matic, J. \& Myerson, A. (2002). Phys. Rev. Lett. 89, 175501.

Gliko, O., Neumaier, N., Pan, W., Haase, I., Fischer, M., Bacher, A., Weinkauf, S. \& Vekilov, P. G. (2005a). J. Am. Chem. Soc. 127, $3433-$ 3438 .

Gliko, O., Neumaier, N., Pan, W., Haase, I., Fischer, M., Bacher, A., Weinkauf, S. \& Vekilov, P. G. (2005b). J. Cryst. Growth, 275, e1409e1416.

Gliko, O., Pan, W., Katsonis, P., Neumaier, N., Galkin, O., Weinkauf, S. \& Vekilov, P. G. (2007). J. Phys. Chem. B, 111, 3106-3114.

Haas, C. \& Drenth, J. (2000). J. Phys. Chem. B, 104, 368-377.

Harano, K., Homma, T., Niimi, Y., Koshino, M., Suenaga, K., Leibler, L. \& Nakamura, E. (2012). Nature Mater. 11, 877-881.

Krishnan, R. \& Lindquist, S. L. (2005). Nature (London), 435, 765-772.

Li, Y., Lubchenko, V. \& Vekilov, P. G. (2011). Rev. Sci. Instrum. 82, 053106.

Li, Y., Lubchenko, V., Vorontsova, M. A., Filobelo, L. \& Vekilov, P. G. (2012). J. Phys. Chem. B, 116, 10657-10664.

Lifshitz, I. M. \& Slyozov, V. V. (1961). J. Phys. Chem. Solids, 19, $35-50$.

Lin, H., Petsev, D. N., Yau, S.-T., Thomas, B. R. \& Vekilov, P. G. (2001). Cryst. Growth Des. 1, 73-79.

Lin, H., Rosenberger, F., Alexander, J. I. D. \& Nadarajah, A. (1995). J. Cryst. Growth, 151, 153-162.

Lomakin, A., Chung, D. S., Benedek, G. B., Kirschner, D. A. \& Teplow, D. B. (1996). Proc. Natl Acad. Sci. USA, 93, 1125-1129.

Lutsko, J. F., Basios, V., Nicolis, G., Kozak, J. J., Sleutel, M. \& Maes, D. (2010). J. Chem. Phys. 132, 035102.

Malkin, A. J. \& McPherson, A. (1994). Acta Cryst. D50, 385-395.

McPherson, A. (2009). Introduction to Macromolecular Crystallography. Hoboken: Wiley-Blackwell.

Nanev, C. N. \& Penkova, A. (2001). J. Cryst. Growth, 232, 285-293.

Nicolis, G. \& Maes, D. (2012). Editors. Kinetics and Thermodynamics of Multistep Nucleation and Self-Assembly in Nanoscale Materials. Hoboken: John Wiley \& Sons.

Ostwald, W. (1896). Lehrbuch der Allgemeinen Chemie. Leipzig: Engelmann.

Pan, W., Galkin, O., Filobelo, L., Nagel, R. L. \& Vekilov, P. G. (2007). Biophys. J. 92, 267-277.

Pan, W., Kolomeisky, A. B. \& Vekilov, P. G. (2005). J. Chem. Phys. 122, 174905.

Pan, W., Vekilov, P. G. \& Lubchenko, V. (2010). J. Phys. Chem. B, 114, 7620-7630.

Pechkova, E. \& Nicolini, C. (2002). J. Cell. Biochem. 85, 243-251. 
Penkova, A., Pan, W., Hodjaoglu, F. V. \& Vekilov, P. G. (2006). Ann. N. Y. Acad. Sci. 1077, 214-231.

Potenza, M. A. C., Sanvito, T., Alaimo, M. D., Degiorgio, V. \& Giglio, M. (2010). Eur. Phys. J. E, 31, 69-72.

Potenza, M., Sanvito, T., Degiorgio, V. \& Giglio, M. (2012). Kinetics and Thermodynamics of Multistep Nucleation and Self-Assembly in Nanoscale Materials, edited by G. Nicolis \& D. Maes, pp. 61-78. Hoboken: John Wiley \& Sons.

Quillin, M. L. \& Matthews, B. W. (2000). Acta Cryst. D56, 791-794.

Reguera, D. \& Rubí, J. M. (2003). J. Chem. Phys. 119, 9888.

Sauter, A., Roosen-Runge, F., Zhang, F., Lotze, G., Jacobs, R. M. J. \& Schreiber, F. (2015). J. Am. Chem. Soc. 137, 1485-1491

Savage, J. R. \& Dinsmore, A. D. (2009). Phys. Rev. Lett. 102, 198302.

Sear, R. P. (2009). J. Chem. Phys. 131, 074702.

Selimović, S., Jia, Y. \& Fraden, S. (2009). Cryst. Growth Des. 9, 18061810.

Shiryayev, A. \& Gunton, J. D. (2004). J. Chem. Phys. 120, 83188326.

Sleutel, M., Lutsko, J., Van Driessche, A. E. S., Durán-Olivencia, M. A. \& Maes, D. (2014). Nature Commun. 5, 5598.

Sleutel, M. \& Van Driessche, A. E. S. (2014). Proc. Natl Acad. Sci. USA, 111, E546-E553.
Sleutel, M., Van Driessche, A. E. S., Pan, W., Reichel, E. K., Maes, D. \& Vekilov, P. G. (2012). J. Phys. Chem. Lett. 3, 1258-1263.

Talanquer, V. (2005). J. Chem. Phys. 122, 084704.

Tsekova, D., Popova, S. \& Nanev, C. (2002). Acta Cryst. D58, 15881592.

Vekilov, P. G. (2004). Cryst. Growth Des. 4, 671-685.

Vekilov, P. G. (2005). J. Cryst. Growth, 275, 65-76.

Vekilov, P. G. (2010a). Cryst. Growth Des. 10, 5007-5019.

Vekilov, P. G. (2010b). Soft Matter, 6, 5254-5272.

Vekilov, P. G., Pan, W., Gliko, O., Katsonis, P. \& Galkin, O. (2008). Aspects of Physical Biology: Biological Water, Protein Solutions, Transport and Replication, edited by G. Franzese \& M. Rubi, pp. 65-95. Heidelberg: Springer.

Vekilov, P. G. \& Vorontsova, M. A. (2014). Acta Cryst. F70, 271-282. Vivarès, D., Kaler, E. W. \& Lenhoff, A. M. (2005). Acta Cryst. D61, 819-825.

Wang, J., Dauter, M., Alkire, R., Joachimiak, A. \& Dauter, Z. (2007). Acta Cryst. D63, 1254-1268.

Wolde, P. R. ten \& Frenkel, D. (1997). Science, 277, 1975-1978.

Yau, S.-T. \& Vekilov, P. G. (2001). J. Am. Chem. Soc. 123, 1080-1089.

Yurkin, M. A. \& Hoekstra, A. G. (2011). J. Quant. Spectrosc. Radiat. Transfer, 112, 2234-2247. 